\title{
IMPLICATIONS OF NRL/ATM SOLAR FLARE OBSERVATIONS ON FLARE THEORIES
}

\author{
C. C. CHENG and D. S. SPICER \\ Naval Research Laboratory, Washington, D.C. U.S.A.
}

Summary. During the Skylab mission, many solar flares were observed with the NRL XUV spectroheliogram in the wavelength region from 150 to $650 \AA$. Because of its high spatial resolution $\left(\sim 2^{\prime \prime}\right)$ the three-dimensional structures of the flare emission regions characterized by temperatures from $10^{4} \mathrm{~K}$ to $20 \times 10^{6} \mathrm{~K}$ can be resolved. Thus the spatial relationship between the relatively cool plasma and the hot plasma components of a flare, and the associated magnetic field structure can be inferred. For example, the Fe xxIV plasma $\left(T \simeq 20 \times 10^{6} \mathrm{~K}\right)$ observed near the soft X-ray maximum during the $2 B$ (M2) disk flare of 1973 , July 15 is elongated perpendicular to the neutral line shown on the photospheric magnetogram, while lines of lower ionization temperatures such as $\mathrm{He}$ II-Fe XVI show the familiar double ribbon structures on either side of the neutral line (Widing and Cheng, 1974). The disk flare of 1973, September 5 shows the same spatial structures. The flare was observed at 1831 UT at the X-ray maximum, and shows that the hot Fe xxIV cloud is located spatially between the ribbons of the He II, Fe XIV-Fe XVI emissions. As the flare cools at 1837 UT, the Fe XxIV cloud disappears while the gap is filled with emissions from ions of lower ionization potentials, and exhibit loop structure. Many other flares also show similar spatial distributions in the XUV emissions. The linear dimensions of the Fe XXIV plasmas as measured from the photospheric plates range from $7000 \mathrm{~km}$ to $14000 \mathrm{~km}$, and the heights of associated loops range from 10000 to $30000 \mathrm{~km}$. From the spatial distributions of the XUV emissions of the many flares, and the comparisons with the magnetograms, we concluded that the magnetic field configuration for the flares we observed are simple bipolar magnetic flux loops with the hot flare plasma located near the top and the cool plasma component on the footprints of the loop.

The implications of these observations are rather clear. Flare models requiring neutral points, neutral lines, or neutral sheets with the exception of Sturrock's inverted $Y$ model, do not seem to agree with the observed magnetic field configuration. Sturrock's model, while rather appealing, appears in conflict with the observational fact that the loops associated with the flares are pre-existing and low-lying. It is hard to form a helmet type field at low altitude, and if this is to occur to a pre-existing loop, the opening of the loop would be the cause of the flare rather than its closing by tearing mode as in Sturrock's model. Elliott's model of high energy particle storage requires large volume and is incapable of explaining the spatial distributions and dimensions of the flare emissions we observed. The Alfvén-Carlqvist current interruption model, is phenomenologically in agreement with our data. However, as has been pointed out by Smith and Priest (1972) the ion-acoustic instability sets in before 
the space charge instability envisioned by Alfvén and Carlqvist. In addition, none of the above models can explain the periodic bursts in XUV, hard X-ray, and microwave often associated with flares. Recently, Cheng and Spicer (1974) proposed a Screw Pinch flare model which invokes the kink and/or the sausage mode instability associated with a simple flux loop. The model is based on the laboratory screw pinch effect and is capable of explaining the periodic bursts and spatial distributions of the hot and cool components of a flare emission. The screw pinch model seems in good agreement with the ATM data. A detailed account of the model will be published elsewhere.

\section{References}

Cheng, C. C. and Spicer, D. S.: 1974, 'A Screw Pinch Flare Model' (preprint, to be published). Smith, D. F. and Priest, E. R.: 1972, Astrophys. J. 131, 213.

Widing, K. G. and Cheng, C. C.: 1974, 'On the Fe xxiv Emission in Solar Flare of June 15, 1973', presented at COSPAR meeting (June 17-July 1), Sao Paulo, Brazil. 